\title{
Triple Mapping to Optimize Axillary Management in Breast Cancer Patients After Neoadjuvant Therapy
}

\author{
Sabrina Shilad, $\mathrm{MD}^{1}{ }^{\mathbb{D}}$, Cagri Cakmakoglu, $\mathrm{MD}^{2}$, Graham Schwarz, $\mathrm{MD}^{2}$, Stephanie Valente, $\mathrm{DO}^{3}$, \\ Risal Djohan, $\mathrm{MD}^{4}$, and Stephen Grobmyer, $\mathrm{MD}^{5}$
}

${ }^{1}$ Department of Breast Services, Cleveland Clinic, Cleveland, OH; ${ }^{2}$ Department of Plastic Surgery, Cleveland Clinic Foundation, Cleveland, $\mathrm{OH} ;{ }^{3}$ Department of Breast Services, Cleveland Clinic Foundation, Cleveland, OH; ${ }^{4}$ Department of Plastic Surgery, Cleveland Clinic, Cleveland, OH; ${ }^{5}$ Department of Surgical Oncology, Cleveland Clinic, Cleveland, OH

\section{INTRODUCTION}

Sentinel lymph node (SLN) biopsy is an option after neoadjuvant chemotherapy in patients who are clinically node negative. False negative rates decrease with dual mapping and identification of more than three SLNs. Patients with positive SLNs require axillary lymph node dissection (ALND). Axillary reverse mapping (ARM) with lymphovenous bypass (LVB) is a lymphedema prevention technique that can be performed in patients who require ALND. It entails injection of isosulfan blue into the proximal extremity to identify lymph nodes and lymphatics draining the arm. LVB performed at the initial surgery reestablishes the drainage of cut lymphatics, potentially decreasing the risk of subsequent lymphedema. We describe a triple mapping technique that can be used to perform both SLN biopsy and subsequent ALND.

\section{METHODS AND RESULTS}

This video introduces a triple mapping technique demonstrating the use of dual-dye technetium-99 and indocyanine green (ICG) for SLN biopsy, and isosulfan blue for ARM.

Electronic supplementary material The online version of this article (https://doi.org/10.1245/s10434-018-6645-9) contains supplementary material, which is available to authorized users.

(C) Society of Surgical Oncology 2018

First Received: 12 June 2018;

Published Online: 13 July 2018

S. Shilad, MD

e-mail: SabrinaShilad@gmail.com
Technetium-99 and ICG are injected periareolarly into the breast for SLN mapping, and isosulfan blue is injected into the proximal extremity for ARM. SLN biopsy is performed, identifying any hot or fluorescent nodes, and ALND is performed if a positive SLN is identified. During ALND, blue lymphatics and nodes are identified, preserved, or marked. LVB re-establishes the continuity of cut lymphatic channels. ICG is then injected into the arm to confirm patency and flow of the new anastomosis.

\section{CONCLUSION}

Triple mapping with ICG, technetium-99, and isosulfan blue represents a novel strategy to optimize axillary management for preventing lymphedema in breast cancer patients following neoadjuvant therapy.

ACKNOWLEDGMENT The authors would like to acknowledge Nikolaus Brown for his video editing. 